\title{
El acuerdo marco de la OMC, ¿modificará los tratados de libre comercio?
}

Del 27 de julio al 1 de agosto de 2004, los 147 países miembros de la Organización Mundial del Comercio firmaron un acuerdo marco, que modificaría sensiblemente el comportamiento prepotente de las potencias económicas y las relaciones asimétricas, que han caracterizado las recientes cumbres de esa organización, así como también las negociaciones desiguales de los tratados de libre comercio y el Área de Libre Comercio de las Américas (ALCA). Los ministros delegados calificaron el acuerdo de Ginebra como "histórico", "un compromiso crucial", un "triunfo del multilateralismo". En cuatro días y medio de tensos debates, contrarreloj, se discutieron y se modificaron temas "tabú" como las masivas subvenciones agrícolas de Europa, Estados Unidos y otras potencias, así como los "temas de Singapur", que paralizaron la "OMC del Desarrollo" de Cancún. Estos fueron algunos de los puntos centrales modificados en el acuerdo marco de Ginebra.

Uno de los actores principales de este acuerdo histórico fue el Grupo de los Veinte (G-20), liderado por Brasil, India, China, que de "grupo subversivo" se convirtió en grupo negociador y logró concesiones importantes de los países del norte. Si se ha hablado de "acuerdo histórico y cambio crucial", algo puede cambiar en la inflexible historia de las cumbres mundiales de la Organización Mundial del Comercio. Y tanto o más importante, algo puede cambiar la misma Organización después de sus repetidos fracasos. Se trata de una euforia prudente.

\section{La prepotencia tradicional de la Organiza- ción}

Terminada la guerra fría y disuelta la Unión Soviética, en 1991, el historiador Lester Thurow publicó Head to Head, traducido al español como La guerra del siglo XXI. La batalla económica que se avecina entre Japón, Europa y los Estados Unidos (E. Vergara, 1992). Aunque los países en desarrollo todavía no aparecen en el subtítulo, se libra una guerra comercial caliente entre la trilogía mayor, en la cual los países emergentes y en desarrollo, al parecer, participarían como "invitados de piedra" en las cumbres de la Organización Mundial del Comercio. A partir de 1992, se inició el proceso de expansión de la gran Europa, que integró a países del antiguo este, en un mercado de $900 \mathrm{mi}$ llones de consumidores, y establecio ta moneda única, en 2001. Japón pasa a ser la segunda potencia mundial y los "tigres asiáticos" se consolidan en un fuerte bloque comercial. Después de quince años de negociaciones tensas, China se integró en la Organización Mundial del Comercio, en la cumbre de Doha (Qatar). En ese mismo año, G. W. Bush relanzó, en Québec, el Área del Libre Comercio de las Américas (ALCA), la cual comprende unos 800 millones de habitantes; pero, a diferencia de los bloques europeo y asiático, integra economías muy desiguales, donde el PIB de Estados Unidos concentra el 79 por ciento del PIB total del resto del continente.

Esto significa que los países pobres y emergentes se verán obligados a librar una doble batalla 
para que sus derechos económicos, sociales y comerciales sean respetados, tanto en las cumbres de la Organización Mundial del Comercio, de inspiración neoliberal, como en los acuerdos bilaterales o regionales de los tratados de libre comercio. No hay que olvidar que Área del Libre Comercio de las Américas, en el fondo, es un proyecto de "América para los americanos" del norte. Ahora podemos decir que el fracaso de la Organización Mundial del Comercio, en Seattle, en 1999, fue una doble victoria. Primero, porque comenzó la ruptura de una larga tradición, según la cual los delegados de los países industrializados presentaban e imponían, a su conveniencia, los temas de la agenda a deliberar; mientras que los representantes de países pobres y emergentes firmaban un documento final, que ni siquiera habían tenido tiempo de leer. Segundo, porque, apoyados por la presencia de unos 50 mil manifestantes pacíficos, los delegados de países pobres y emergentes protestaron - entre otras cosas - por las masivas subvenciones agrícolas (300 mil millones de dólares al año; mil millones diarios) que Europa, Estados Unidos, Japón y otros... otorgan a sus agricultores y agro exportadores. De acuerdo con las cláusulas de la Organización Mundial del Comercio, sus acuerdos fil ales deben ser firmados por todos los estados miémbros. Los delegados de los países pobres y emergentes se negaron a firmar un acuerdo que desconocían y a ser tratados como simples invitados de piedra. El fracaso de Seattle fue una victoria, tanto por la firme postura de estos delegados como por el fortalecimiento de la presencia activa de los manifestantes pacíficos, en todas las cumbres mundiales. Esta fue la semilla del foro social mundial de Porto Alegre.

Las esperanzas estaban puestas en la cumbre de la Organización Mundial del Comercio de Doha (2001). Pero esa fecha no era la más propicia. A dos meses del 11 septiembre, los temores y problemas del tercer mundo podían quedar relegados a un segundo plano. Tal como dijera Rigoberta Menchú: "El dolor del pueblo norteamericano ha opacado el dolor de todos los pueblos que sufren". En la reunión de Doha se firmó una serie de compromisos, entre ellos: "la reducción progresiva de las subvenciones y otros apoyos a las agriculturas, con el fin de llegar a su progresiva reducción", algunas promesas sobre medicamentos genéricos y pocas cosas más. Sin embargo, después de Qatar, estos compromisos se convirtieron en palabras huecas: las subvenciones masivas fueron más masivas (Realidad, 2002, 85, pp. 12-19).
La cumbre de "la OMC del desarrollo", en Cancún (2003), fue novedosa. Además de la presencia multitudinaria de manifestantes pacíficos mexicanos, surcoreanos, africanos, etc., los países pobres y emergentes se habían organizado en el Grupo de los Veinte (G-20), liderados por los ministros de Brasil, India y China. Este grupo dijo que no se podía avanzar sin tratar, en primer lugar, las masivas subvenciones agrícolas. Así se lo manifestó Lula da Silva a G. W. Bush (ECA, 2003, pp. 1060-1070). Los representantes de Estados Unidos, R. Zoellick, y de Europa, P. Lamy, lanzaron una pregunta: ¿qué nos ofrecen ustedes para que nosotros les ofrezcamos algo? Lo primero que debía discutirse era la agenda de los "temas de Singapur" (1996) - inversiones, libre competencia, servicios públicos, derechos de propiedad intelectual-. Una vez más, a ejemplo de Seattle, los representantes de los países emergentes y en desarrollo se negaron a firmar el acuerdo final con las naciones del norte, las cuales habían incumplido los compromisos pactados en Qatar. La Organización Mundial del Comercio se declaró, entonces, incapaz de cumplir la misión que le había sido confiada: programar acuerdos equitativos, aceptables por los representantes de todos los países. De hecho, las "mismas manzanas de la discordia" que hicieron fracasar Cancún, son las que se quieren enmendar y corregir en el acuerdo marco de la reciente cumbre de Ginebra.

El fracaso de Cancún está resumido en los titulares de algunos diarios. "El enfrentamiento por el mercado agrícola paraliza la cumbre de la OMC" (El País, 13 de septiembre de 2003). "En Cancún se abre la gran batalla del comercio mundial" (Le Monde, 9 de septiembre de 2003). "En Cancún los bloques comerciales se miran de arriba abajo y se organiza la confrontación" (Le Monde, 10 de septiembre de 2003). "Las subvenciones agrícolas en el centro del debate en la cumbre de la OMC" (Le Monde, 12 de septiembre de 2003). "Las negociaciones se estancan en Cancún" (Le Monde, 14 de septiembre de 2003). "Finaliza la cumbre de Cancún con un fracaso absoluto de las negociaciones" (El País, 14 de septiembre de 2003). "Los grandes desacuerdos norte-sur llevan al fracaso de la OMC" (Le Monde, 15 de septiembre de 2003). "Países ricos intentan dividir a grupos antisubsidios" (TIMSN, 12 de septiembre de 2003).

A la salida de la conferencia de Cancún, $\mathbf{R}$. Zoellick declaró con tranquilidad: "La estrategia comercial de Estados Unidos avanza en varios fren- 
tes: tenemos acuerdos bilaterales con seis países. Negociaremos con los otros catorce". En este escenario de prepotencia se negociaron, sucesivamente, el tratado de libre comercio de Centroamérica con Estados Unidos y, a finales de noviembre, el Área de Libre Comercio de las Américas, en plural, donde se enfrentaron el norte y el sur, R. Zoellick, por Estados Unidos, y Celso Amorín, por MERCOSUR. El ambiente del tratado de libre comercio de Centroamérica se puede resumir en algunos titulares. "Máxima autoridad comercial norteamericana llega al país. TLC con EUA bajo la lupa de Zoellick. Jalón de orejas por OMC" (La Prensa Gráfica, 29 de septiembre de 2003). "Zoellick toma el mando del TLC" (El Diario de Hoy, 29 de septiembre de 2003). "Costa Rica puede quedar fuera del TLC. E.U.A. exige abrir telecomunicaciones. Zoellick da instrucciones a región para negociar" (La Prensa Gráfica, 2 de octubre de 2003). "Estados Unidos exigen poner todo el agro sobre la mesa", "Transcienden rumores de que El Salvador intentó lanzar propuestas agrícolas negativas para la región" (La Prensa Gráfica, 3 de octubre de 2003). "Zoellick exige abrir el agro. Nosotros no presionamos, negociamos" ( $E l$ Diario de Hoy, 3 de octubre de 2003). Falta agregar que los puntos espinosos, relacionados con los "temas de Singapur" se reservaron para la última reunión, la cual tuvo lugar en Washington.

\section{La cumbre de Miami y la Declaración Mi- nisterial del ALCA}

La sombra de Cancún se proyectó sobre la reunión de Miami para afinar las cláusulas finales del ALCA, el cual debía comenzar en enero de 2005. "Tratando de evitar confrontaciones que generaron el fiasco de la OMC en Cancún, los dos copresidentes de las negociaciones, Estados Unidos y Brasil, redujeron el tono de la confrontación. Antes de ir a Miami, elaboraron un 'acuerdo a la carta', que ha recibido el aval de los ministros de Comercio de las Américas, adelantando así el cierre de la conferencia. El acuerdo permite a cada país signatario no adherirse a las cláusulas del ALCA que estime contrarios a sus intereses... El acuerdo permite seguir avanzando, dejando de lado los asuntos más espinosos, como las subvenciones agrícolas".

Una de las principales diferencias entre las negociaciones bilaterales del tratado de Centroamérica y del proceso pactado en el ALCA es que, debido a las presiones del MERCOSUR, en este segundo caso se crearon ocho grupos para negociar "temas espinosos" y tres cuerpos consultivos, al frente de los cuales se colocaron presidentes y vicepresidentes de los diferentes países signatarios. "Grupos de negociaciones del ALCA: grupo de negociaciones sobre el acceso a los mercados; sobre la agricultura; sobre las inversiones; sobre la política de competencia; sobre los derechos de propiedad intelectual; sobre los servicios; sobre el reglamento de diferendos; sobre las subvenciones, los derechos de antidumping y derechos compensadores. Además se han creado tres 'grupos auxiliares': el grupo consultivo sobre economías pequeñas, el comité de representantes gubernamentales sobre participación de la sociedad civil, y el comité técnico sobre cuestiones institucionales" (Le Monde, 20 de noviembre de 2003). La Declaración Ministerial de Miami contiene un preámbulo de lo que puede ser el acuerdo marco de Ginebra ("Un ALCA a dos velocidades", ECA, 2004, pp. 145-152).

\section{La voz de las iglesias}

Entre el 10 y el 13 de julio de 2003, un grupo de laicos y laicas, religiosas y jesuitas dedicados a la promoción social se reunieron en Quito, para discutir su preocupación y rechazo al proyecto del ALCA. Los días 2-4 de septiembre, los obispos representantes de las conferencias episcopales de los países del MERCOSUR, Chile y Bolivia se reunirian en Montevideo para reflexionar sobre algunos desafíos éticos y pastorales que supondría la implementación de este proyecto. En razón de la brevedad, tomo algunas ideas de ambos documentos, los cuales se aplican al tratado de libre comercio centroamericano.

El ALCA y el futuro de los pueblos. Por qué no queremos el ALCA. Porque la composición del ALCA es impresionantemente asimétrica... Del PIB total, el 79 por ciento corresponde a Estados Unidos; el 5,9 por ciento a Canadá; el 4,7 por ciento a Brasil; a México, el 4,2 ciento; a Argentina, el 2,51 por ciento y la gran mayoría de países no supera el 1 por ciento del PIB total del ALCA. Por lo tanto, la negociación del ALCA se realiza en términos de poderes extremadamente desiguales y asimétricos. Porque la forma de negociación del ALCA no es transparente. La negociación sigue siendo un secreto y el texto no logra incorporar las diversas propuestas que han presentado organizaciones ciudadanas del continente. En la mayor parte de los países, la sociedad civil se encuentra sin información sobre lo que sus gobiemos están negociando. 


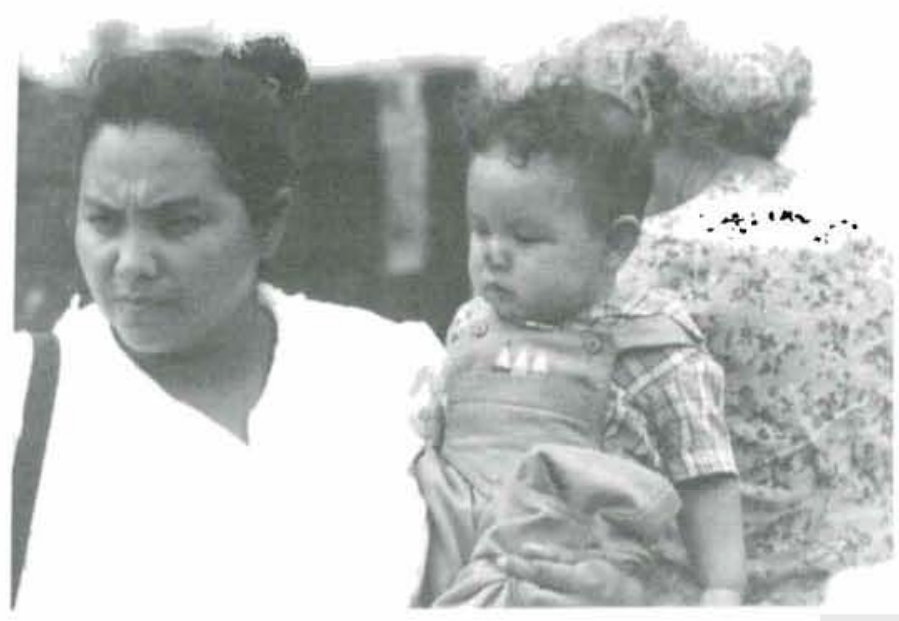

países, algunos de ellos con economías muy fuertes y desarrolladas, otros muy débiles, y el desequilibrio de intereses y poderes, podrían traer consecuencias muy graves, especialmente en relación a la identidad cultural, los puestos de trabajo y la misma subsistencia de las economías más frágiles. Más que de una integración podría tratarse de un neocolonialismo con un impacto negativo sobre las comunidades locales.

Este proyecto tendería a propiciar una concentración del poder económico en pocas manos y en pocas empresas competitivas, favoreciendo la formación de monopolios y oligopolios, que terminarían por imponer su hegemonía

Porque el ALCA es mucho más que un tratado comercial. Es un esquema de privatización de servicios y bienes públicos, tradicionalmente reservados al Estado, de liberación de mercados y de seguridad para las inversiones extranjeras, a las que se conceden ventajas sobre las nacionales. Las propuestas del ALCA van a mermar la facultad de los gobiemos de llevar a cabo políticas de desarrollo específicas, en áreas económicas y sociales. Se pretende que el ALCA tenga un rango supraconstitucional. Con ello se limitará la soberanía de las naciones que firmen el tratado... Queremos un nugvo tipo de integración de las Américas. Teóricamente, hay otras posibilidades $\mathrm{y}$, empíricamente, hay diversas formas de integración de los distintos países y bloques, distintos de la dinámica globalizada. Se está consensuando una alternativa para las Américas. Otra integración es posible.

No ignoramos la complejidad y las diversas percepciones que suscita en la sociedad. Por eso pensamos que es necesario no solo un análisis técnico-económico, sino también ético-social de la cuestión. Nos preocupa, en primer lugar, la insuficiente información y la rapidez con la que se impulsa dicho proyecto, con la consiguiente falta de participación de todos los sectores de la sociedad civil, en una toma de decisión tan importante para el futuro de nuestros pueblos. Una eventual integración de los países americanos debería tener en cuenta no solo el aspecto económico y comercial, sino todas las dimensiones de la persona humana: sociales, políticas, culturales, religiosas. La excesiva desproporción de las capacidades competitivas entre nuestros a los gobiernos, especialmente en los países más débiles del continente americano. Un proyecto del ALCA debería tener como primera motivación la promoción del bien común y de la solidaridad entre los pueblos y no la búsqueda del mayor provecho de algunos pocos y de los más poderosos en detrimento de los más débiles.

Puede parecer que la implementación del ALCA es irreversible. Sin embargo, es también posible cambiar algunos de los términos para que los países en vías de desarrollo tengan preferencias competitivas, se respete su soberanía y autodeterminación y sus recursos naturales estratégicos no sean susceptibles de apropiación privada. En este sentido, es importante reconocer y participar en la construcción y fortalecimiento de bloques regionales y subregionales, en nuestro continente. Un verdadero proceso de integración en América debe basarse en una política continental, que tenga en cuenta los derechos humanos y los principios de soberanía, la justicia, la solidaridad y el respeto a las identidades culturales de los pueblos. Una vez más afirmamos, inspirados en la doctrina social de la Iglesia, que la economía debe estar al servicio de la persona humana y respetar los derechos fundamentales de los pueblos.

\section{El puente Miami-Ginebra}

Al relacionar la cumbre del ALCA de Miami y la de la Organización Mundial del Comercio de Ginebra conviene recordar que la actividad del G-20 y, es especial de Brasil, jefe de fila del MERCOSUR, ha sido muy intensa en los prime- 
ros meses de 2004. En enero tuvo lugar el foro social de Bombay y el foro económico de Davos, donde saltó la crítica a la Organización Mundial del Comercio. Al terminar el año, en Montevideo, tuvo lugar una cumbre del MERCOSUR, a la cual asistieron Pascal Lamy, representante de la Unión Europea, Igor Ivanov, Ministro de Asuntos exteriores de Rusia, y Fernando Dias do Santos, Primer Ministro de Angola. Aparte que su presencia dio realce a la cumbre, estos invitados especiales "respaldaron la voluntad del bloque suramericano de diversificar sus relaciones comerciales a través de acuerdos con la Europa comunitaria y extracomunitaria y con la Unión Africana".

Si en la cumbre de Miami, R. Zoellick quiso aislar a Brasil, para lo cual ofreció una serie de tratados de libre comercio a varios países suramericanos; ahora es Brasil el que ofrece un acuerdo regional a estos mismos países, más cercanos geográfica y culturalmente. "La cumbre aprobó el ingreso de Perú como Estado asociado del MERCOSUR, condición que ya tienen Chile y Bolivia, y firmó un acuerdo de complementación económica con Bolivia, Colombia, Ecuador, Perú y Venezuela (CAN), que coloca bajo el mismo paraguas a 10 de las 13 naciones de América del Sur. El acuerdo, estiman los firmantes, representa un paso importante hacia la creación de una zona de libre comercio, en un espacio de 340 millones de consumidores y de cerca de un billón de dólares de PIB. El poder de este bloque debería reflejarse a la hora de aunar criterios en las negociaciones con las potencias comerciales".

De esta manera, se genera un poder compensatorio, luego del fracaso de la cumbre de Cancún; asimismo, se alcanzó un acuerdo en más del 90 por ciento de las diferencias comerciales entre el MERCOSUR y la Comunidad Andina. "Este es un hecho notable, que cambia toda la geografía económica y comercial de la región", dijo Celso Amorín. "La cumbre también ha reconocido las asimetrías o desproporciones entre los dos socios mayores (Brasil y Argentina) en relación con Paraguay y Uruguay. Se ha creado un 'fondo de compensación' para las economías más débiles, siguiendo las experiencias de la Unión Europea". El Banco Brasileño de Desarrollo "podría ayudar a reabsorber estos desequilibrios por medio de créditos como los ya concedidos a Ecuador y Venezuela", admitiendo la posibilidad de "tiempos diferentes" para llegar a una unión.

Los representantes de Brasil hablaron también de un parlamento, elegido por sufragio universal y de una moneda común. La cumbre ha decidido crear la nueva comisión de representantes permanentes de MERCOSUR. Al mismo tiempo que diseña las normas básicas comerciales e institucionales para integrarlo y fortalecer la alianza con la Comunidad Andina de Naciones. La Unión Europea y MERCOSUR deben concluir un acuerdo de libre comercio entre los dos bloques, a finales de 2004. Igualmente, se estrecharon las relaciones comerciales con la Europa extra comunitaria y la Unión Africana (El País, 18 de diciembre de 2003; Le Monde, 18 de diciembre de 2003). Toda esta serie de actividades, lideradas por Brasil, en orden a ampliar acuerdos comerciales continentales y extra continentales, pueden servimos de puente para entender el papel negociador de Celso Amorin, en la cumbre de la Organización Mundial del Comercio de Ginebra. Cuando ésta se hallaba en estado de hibernación, este activismo comercial puede leerse como el deseo de pasar del bilateralismo al multilateralismo comercial.

\section{El acuerdo marco de Ginebra}

La editorial de El País, "Compromiso crucial", afirma que "la OMC ha logrado evitar un desastre, al acordar en Ginebra, contrarreloj y en el último minuto, un compromiso que abre el camino a la reactivación del comercio, mediante el recorte de las subvenciones agrícolas en los estados más ricos. El fracaso de las negociaciones hubiera constituido una seria amenaza al crecimiento de la ya desestabilizada economía mundial. La ruptura con que se saldó la reunión ministerial de Cancún, en septiembre del pasado año, ilustró la hipocresía y el doble lenguaje que pueden llegar a exhibir los gobiemos de las principales economías, cuando se trata de defender los intereses de pequeños grupos, a costa de la prosperidad de la mayoría y de la estabilidad del propio sistema de relaciones comerciales internacionales. A la persistencia de las ayudas agrícolas en las economías ricas, estimadas en más de 250.000 millones de euros anuales, se opusieron allí de forma resulta un amplio grupo de países en desarrollo, liderados por Brasil, paralizando la ronda de negociaciones liberalizadoras en curso".

El negociador europeo, Pascal Lamy, se comprometió, el pasado mes de mayo, a reducir los subsidios a la exportación de productos agrícolas, dando así un paso para que Estados Unidos procediera también a reducir su ayuda a los agricultores. "Pero las ventajas de la recuperación de la 
credibilidad de la OMC pueden ser mucho mayores, si los avances se extienden a otros bienes y servicios, contemplados en la ronda de negociaciones iniciada en Doha hace tres años. Estimaciones del Banco Mundial sitúan en 250000 millones de dólares (a precios de 1997) las ganancias globales derivadas del libre comercio a partir de 2015 , con una parte importante de las mismas localizadas en las economías emergentes. La reducción de la pobreza derivada de la extensión de esas ganancias a un número cada vez mayor de países sería la demostración más elocuente de que la globalización en curso no es una forma de dominación de los grandes. Constituiría también la credencial más importante para la más joven de las instituciones internacionales, la OMC, que ahora en Ginebra ha vuelto a respirar" (El País, 2 de agosto de 2004).

Los corresponsales de Le Monde comienzan diciendo que el acuerdo de Ginebra ha sido un "triunfo del multilateralismo", al romperse el tabú de las masivas subvenciones agrícolas. La euforia de los principales actores del acuerdo es normal: "Lo hemos logrado, lo cual prueba que, cuando hay voluntad política, todo es posible. El multilateralismo ha logrado una victoria, dice el director general de la OMC, y el día que concluyamos el ciclo de Doha será un triunfo". Pascal Lamy se felicita "porque de nuevo el ciclo de Doha se ha encarrilado. Hemos cubierto el $50 \%$ del camino y el acuerdo de Ginebra es bueno para Europa, es bueno para los países en desarrollo, es bueno para todo el mundo". También R. Zoellick muestra su satisfacción: "tenemos un plano que marca el camino a seguir; desde septiembre vamos a negociar los límites de velocidad, fijar la manera y el ritmo a que reduzcamos las barreras arancelarias". "Todo se ha hecho por consenso, agregó Zoellick; si los países en desarrollo han aceptado el acuerdo, no es por filantropía ni debido a presiones; simplemente por ser en interés de todos".

Otros testimonios marcan la mitad del camino. Celso Amorín, quien ha tenido un papel importante en estas negociaciones, dice que el acuerdo "es el comienzo del fin de las subvenciones". Su colega japonés comenta que, después de Cancún, el G-20 "era considerado como un grupo subversivo, pero después de Ginebra ha demostrado su poder negociador y de lograr concesiones de parte de los países del norte". El embajador de China en la Organización Mundial del Comercio cree que "de una manera general, el acuerdo no es malo, aunque los países en desarrollo no están totalmente contentos". El delegado de Australia insiste en la dificultad del camino que queda por recorrer, aspecto en el cual insiste el ministro francés. Los representantes de África Occidental muestran su alegría, si Estados Unidos procede a reducir sus subvenciones al algodón (no hay una fecha concreta, ni un compromiso claro), porque ello mejoraría la suerte de sus agricultores (Le Monde, 2 de agosto de 2004). Esta serie de testimonios muestra los claroscuros de un acuerdo que, visto desde la historia pasada de la Organización Mundial del Comercio, significa un triunfo, pero también, visto desde la misma historia, también significa un camino difícil de recorrer.

\section{Los principales puntos del acuerdo de Gine- bra}

Las primeras reformas se centran en la "Agricultura". El acuerdo busca dar a los productos agrícolas de los países en desarrollo una mejor oportunidad para competir con los productos de países desarrollados. "Competencia en la exportación", y a este fin se quieren corregir tres dispositivos, criticados por los países pobres: "las subvenciones a la exportación (utilizadas sobre todo por la Unión Europea); los créditos a la exportación (utilizados sobre todo por Estados Unidos) y los monopolios de exportación de empresas comerciales de Estado (casos de Canadá y Australia). "Todos los países miembros de la OMC tendrán que aplicar los mismos recortes, una vez entre en marcha el acuerdo, para el que todavía no hay una fecha establecida". La única excepción son los 50 países más pobres de la Organización, que no tendrán que imponer esta medida.

El primer paso es una reducción del 20 por ciento del total de los subsidios, en el primer año de vigencia del acuerdo. Además, el esquema establecido prevé que los productos agrícolas que reciben más ayuda experimenten los recortes principales. Este sistema también se aplicará a los "aranceles" que dificultan la entrada de productos agrícolas de otros países: los mayores recortes los experimentarán los productos más protegidos. En contrapartida, los países desarrollados han obtenido la facultad de mantener derechos de aduana elevados para algunos productos "más sensibles" (por ejemplo: el arroz, en Japón; la leche, el azúcar y la carne bovina, en Europa). Incluso para estos productos, los países deben comprometerse a abrir más sus mercados, sea por reducción de aranceles, sea por eleva- 
ción de cuotas de importación. Los países miembros deben definir cuáles son sus "productos sensibles". "El país que más ha tenido que ceder ha sido Estados Unidos, que tendrá que igualar los recortes prometidos por Europa. Estados Unidos tiene que recortar parte de lo 1900 millones de dólares de ayudas al algodón, azúcar y trigo entre otros".

El acuerdo en el apartado de los "Servicios", aunque de gran importancia para los países ricos, sigue siendo ambiguo. Estos países desean liberalizar el sector. Para ello, el acuerdo prevé que cada país miembro haga sus ofertas, es decir, que informe a los otros qué servicios está dispuesto a liberalizar. El texto precisa que las ofertas concretas deben ser presentadas en mayo de 2005. Respecto a las "trabas aduaneras", el texto insiste en una reducción de los obstáculos burocráticos, que entorpecen el tráfico de mercancías en las fronteras. Los 147 países miembros han pactado ayudar a que los países más pobres no se vean afectados negativamente por ninguna medida de la Organización Mundial del Comercio. Los países más desarrollados piden, sobre todo a los países emergentes, reducir los aranceles con que se protegen de los bienes industriales producidos en el norte. A cambio de las reducciones en las ayudas agrícolas, los países emergentes se han comprometido a recortar sus aranceles industriales, "aunque todavía no se han asignado cifras, ni objetivos específicos". El esquema será parecido al de las ayudas agrícolas, reducir más los aranceles en los bienes industriales más protegidos.

En relación con los "Temas de Singapur", los países desarrollados han aceptado dejar de lado tres de los cuatro temas adoptados en esa reunión, en 1996: mercados públicos, inversiones y competencia. El texto propone abrir negociaciones sobre el cuarto tema: "facilitación de los intercambios", es decir, simplificación de trámites aduaneros. ("El comercio mundial se prepara para poner fin a los subsidios a la agricultura", El País, 2 de agosto de 2004; "Les principaux points de l'accord-cadre de Genéve", Le Monde, 1 de agosto de 2004; "Quatre jours et une nuit pour tenter de relancer le cycle de Doha, et une France isolée", Le Monde, 1 de agosto de 2004.)
Este acuerdo marco, gestado y firmado contrarreloj, crea una euforia prudente: algo ha cambiado y algo parece puede cambiar. Por un lado, un nuevo fracaso en Ginebra, después del fiasco de Cancún, hubiera significado la quiebra de la Organización Mundial del Comercio (la institución internacional más joven) y, en consecuencia, la quiebra del orden comercial mundial, en un momento en que esa economía está bien desequilibrada. La pérdida de credibilidad se extendería al mismo modelo de libre mercado, cuando a la inseguridad económica de los países pobres se suma la inseguridad física y psíquica de los países ricos. Lo que ha movido a los gobiemos de los países ricos a hacer estas concesiones, ¿es atrición o es contrición? Es decir, ¿es miedo a que "la globalización de la ira se acelere ante el rápido crecimiento de las desigualdades" o es el propósito de caminar hacia un mundo donde haya mayor seguridad y prosperidad para todos, gracias a la cooperación de todos? De esto se habló en el foro de Davos de 2004.

Por otra parte, los negociadores son representantes de los gobiemos, sometidos a la fuerte presión de cumplir con un calendario. Las grandes empresas y los grupos de poder económicos organizados, ¿aceptarán las nuevas cláusulas del acuerdo y se conformarán, en forma decente y transparente, con la mitad del camino por recorrer? La contraparte es que el Grupo de los Veinte ha dejado de ser un "grupo subversivo" para ser un "grupo negociador", que ha logrado grandes concesiones de los países poderosos. Son muchos los claroscuros. Pascal Lamy, dicen, ya no será el negociador de la Unión Europea, y la suerte de Robert Zoellick depende del resultado de las elecciones presidenciales de Estados Unidos. Si la Organización Mundial del Comercio vuelve a respirar dentro de este acuerdo marco, los latidos se harán sentir en los enfoques y contenidos de los tratados de libre comercio y del ALCA, tema de preocupación en toda la América Latina. Los años 2004 y 2005 serán años de esperanza y de euforia prudentes.

F. JAVIER IBISATE $S$. J. Catedrático del Departamento de Economía de la UCA 